ISSN 0258-7122

Bangladesh J. Agril. Res. 36(1) : 1-12, March 2011

\title{
EFFECT OF REMOVAL OF SOME PHOTOSYNTHETIC ORGANS ON YIELD COMPONENTS IN DURUM WHEAT (Triticum aestivum L.)
}

\author{
ALPAY BALKAN ${ }^{1}$, TEMEL GENÇTAN ${ }^{2}$ AND OĞUZ BILGIN ${ }^{3}$
}

\begin{abstract}
This research was carried out in experimental field of Field Crops Department of Agricultural Faculty of Namik Kemal University in randomized split block design with three replications per treatment during 2004-2005 and 2005-2006. The objective of this study was to find out the contribution rates of awn, flag leaf, $1^{\text {st }}$ upper leaf blade, $2^{\text {nd }}$ upper leaf blade and other leaf blades to main yield components in three durum wheat cultivars (cv. Kiziltan-91, Kunduru-1149, and Yelken-2000). The results of this experiment showed that removal of awn, flag leaf, $1^{\text {st }}$ upper leaf blade, $2^{\text {nd }}$ upper leaf blade, and other leaf blades reduced significantly spike weight, number of grains per spike, grain weight per spike, and 1000-grain weight except the number of spikelets per spike. It was concluded that the organs play an important role in grain yield in durum wheat during grain filling stage.
\end{abstract}

Keywords: Photosynthetic organs, yield components, durum wheat.

\section{Introduction}

Products of photosynthesis are highly used in grain filling after heading (Thorne, 1973). Contribution of different photosynthetic organs to grain yield reduces with distance of these organs from grain. The most of photosynthetic products are obtained from upper photosynthetic organs of wheat plant during grain filling period (Austin and Jones, 1975 Mahmood and Chowdhry, 1997). In addition, ears of cool season cereals are photosynthetically active and play an important role in providing saccharides during grain filling (Jiang et al., 2006).

During the grain filling period, awns, flag leaf, first upper leaf, second upper leaf and third upper leaf are the potentially efficient photosynthetic organs in terms of economic production in wheat (Mahmood and Chowdhry, 1997). Contribution rate of flag leaf to daily photosynthetic products varies from $50 \%$ to $60 \%$. However, contribution rate of ear to grain yield is associated with ear type (awned ear or awnless ear; compact ear or sparse ear). Contribution of glumes and awns to grain yield can be very high depending on environmental conditions (Evans and Rawson, 1970; Birsin, 2005). Photosynthetic activity of ear depends on the release of $\mathrm{CO}_{2}$ as result of respiration of grains (Migus and Hunt, 1980).

${ }^{1}$ Res. Asstt., Namik Kemal University, Agricultural Faculty, Field Crops Department, Tekirdağ/Turkey, ${ }^{2}$ Prof. Namik Kemal University, Agricultural Faculty, Field Crops Department. Tekirdağ/Turkey, ${ }^{3}$ Asstt. Prof., Namik Kemal University. Agricultural Faculty, Field Crops Department, Tekirdağ/Turkey. 
Awns are very close to grains and their photosynthetic activity is very high. Besides, they have a xeromorphic characteristic. Thus, awns may serve as a high photosynthetic organ during periods of drought stress and actually compensate for flag leaf photosynthesis reduced by drought stress (Genc, 1977; Koc et al., 1985; Weyhrich et al., 1995). Therefore, awned cultivars of cool season cereals have more stable yields under warm and dry climatic conditions (Jiang et al., 2006).

The main objective of this experiment was to determine the effects of the removal of main photosynthetic organs on the yield components, such as spike weight, number of spikelets per spike, number of grains per spike, grain weight per spike, and 1000-grain weight of durum wheat cultivars under the agroclimatic conditions of Tekirda during 2004-2005 and 2005-2006.

\section{Materials and Method}

An experiment was conducted at Experimental Field of Agricultural Faculty, Namik Kemal University in Tekirda, Turkey at latitude $40^{\circ} 36^{\prime}-40^{\circ} 31^{\prime}$ and longitude $26^{\circ} 43^{\prime}-28^{\circ} 08^{\prime}$ and altitude $10 \mathrm{~m}$ during 2004-2005 and 2005-2006 growing seasons. The soil texture was slightly acidic ( $\mathrm{pH}$ 6.5), saltless $(0.04 \%)$, low in lime $(0.63 \%)$ and organic matter $(1.66 \%)$ content with adequate potassium and phosphorus. Climatic data during the growing period are shown in Table 1 below:

Table 1. The climatic data of the experimental area during the growing periods.

\begin{tabular}{c|ccc|c|c|c}
\hline \multirow{2}{*}{ Months } & \multicolumn{2}{|c|}{ Rainfall $(\mathrm{mm})$} & \multicolumn{2}{c|}{ Temperature $\left({ }^{\circ} \mathrm{C}\right)$} & \multicolumn{2}{c}{ Relative humidity (\%) } \\
\cline { 2 - 7 } & $2004-2005$ & $2005-2006$ & $2004-2005$ & $2005-2006$ & $2004-2005$ & $2005-2006$ \\
\hline November & 27.7 & 105.2 & 11.4 & 9.7 & 80.8 & 82.4 \\
December & 45.5 & 91.2 & 7.7 & 7.3 & 84.0 & 83.1 \\
January & 62.7 & 26.2 & 6.1 & 2.4 & 84.0 & 83.0 \\
February & 74.9 & 76.9 & 4.2 & 4.4 & 84.0 & 86.8 \\
March & 20.9 & 101.6 & 7.6 & 8.0 & 74.1 & 87.9 \\
April & 12.7 & 9.5 & 12.2 & 12.4 & 76.3 & 82.9 \\
May & 78.2 & 14.1 & 16.9 & 17.2 & 76.2 & 81.1 \\
June & 13.0 & 29.0 & 20.5 & 21.6 & 76.5 & 78.0 \\
\hline
\end{tabular}

The experiment was laid out in a randomized split block design with three replications. Durum wheat cultivars viz. Kiziltan-91, Kunduru-1149, and Yelken2000 were used in the experiment. Each cultivar was sown in a main plot $\left(5 \mathrm{~m}^{2}\right)$ 
consisting of 6 rows. The seeding rate was 500 seeds per square meter of plot. The cultivars were planted in mid November each year. A total of $130 \mathrm{~kg} \mathrm{~N} / \mathrm{ha}$ and $50 \mathrm{~kg} \mathrm{P} / \mathrm{ha}$ were applied at sowing, tillering, and preanthesis periods in both years.

Following treatments were used and data were collected from 10 randomly selected plants at heading stage (59 stage of Zadoks scale):

$\mathrm{T}_{1}=$ Control (intact plants)

$\mathrm{T}_{2}=$ All leaf blades removed

$\mathrm{T}_{3}=$ All leaf blades and awns removed

$\mathrm{T}_{4}=$ Except flag leaf blade, other leaf blades removed

$\mathrm{T}_{5}=$ Except flag leaf blade and 1st upper leaf blade, other leaf blades removed

$\mathrm{T}_{6}=$ Except flag leaf blade, 1 upper leaf blade and 2nd upper leaf blade, other leaf blades removed

$\mathrm{T}_{7}=$ Only awns removed

$\mathrm{T}_{8}=$ Only flag leaf blade removed

$\mathrm{T}_{9}=$ Flag leaf blade and 1st upper leaf blade removed

$\mathrm{T}_{10}=$ Flag leaf blade, 1 upper leaf blade and 2nd upper leaf blade removed

Data were recorded from sample plants on spike weight, number of spikelet per spike, number of grains per spike, grain weight per spike and 1000-grain weight at maturity (92. stage of Zadoks scale). Data so collected were analyzed by using the analysis of variance techniques (Steel and Torrie, 1984) and Least Significant Different (LSD) Test was used to see the significance of treatment means (Düzgüneş et al., 1987).

\section{Results}

Effects of 10 different treatments on spike weight, number of spikelets per spike, number of grains per spike, grain weight per spike, and 1000-grain weight are given in Table 2 and 3.

The results show that the spike weight was $3.54 \mathrm{~g}$ in 2005 and $2.95 \mathrm{~g}$ in 2006. Number of spikelets per spike was not statistically significant among the treatments, it was 22.75 in 2005 and 22.42 in 2006 . Number of grains per spike was 49.97 in 2005 and 48.63 in 2006. Grain weight per spike was $2.79 \mathrm{~g}$ in 2005 and $2.35 \mathrm{~g}$ in 2006. Thousand-grain weight was $50.47 \mathrm{~g}$ in 2005 and $49.56 \mathrm{~g}$ in 2006 . At heading stage, the crop received more rains, especially in May 2005 than in May 2006 (Table 1). Therefore, mean values of yield components obtained from 2004-2005 growing period were higher than those of 2005-2006 growing period. 
Table 2. Yield components of wheat as affected by the removal of some photosynthetic organs in 2005.

\begin{tabular}{|c|c|c|c|c|c|c|c|c|c|c|c|c|c|c|c|c|c|c|c|c|}
\hline \multicolumn{5}{|c|}{ Spike weight (g) } & \multicolumn{4}{|c|}{ Number of spikelet per spike } & \multicolumn{4}{|c|}{ Number of grain per spike } & \multicolumn{4}{|c|}{ Grains weight per spike (g) } & \multicolumn{4}{|c|}{ 1000-grain weight (g) } \\
\hline \multirow[b]{2}{*}{ 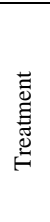 } & \multicolumn{3}{|c|}{ Cultivars } & \multirow[b]{2}{*}{$\stackrel{\mathbb{\Xi}}{\Sigma}^{\mathbb{N}}$} & \multicolumn{3}{|c|}{ Cultivars } & \multirow[b]{2}{*}{$\stackrel{\mathbb{\varpi}}{\Sigma}^{\tilde{\Xi}}$} & \multicolumn{3}{|c|}{ Cultivars } & \multirow[b]{2}{*}{$\begin{array}{l}\mathbb{\Xi}^{\Xi} \\
\sum\end{array}$} & \multicolumn{3}{|c|}{ Cultivars } & \multirow[b]{2}{*}{$\sum_{\Sigma}^{\bar{\Xi}}$} & \multicolumn{3}{|c|}{ Cultivars } & \\
\hline & 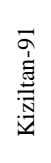 & 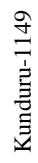 & 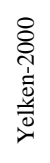 & & 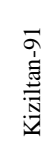 & 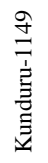 & 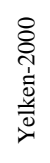 & & 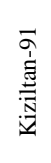 & 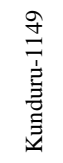 & 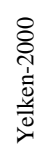 & & 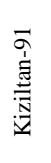 & 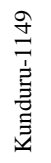 & 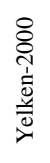 & & 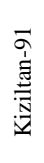 & 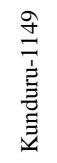 & 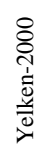 & 茄 \\
\hline
\end{tabular}

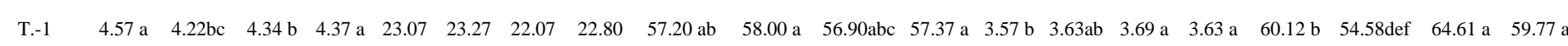

$\begin{array}{llllllllllllllllllllll}\text { T.-2 } & 2.71 \mathrm{r} & 3.06 \mathrm{op} & 2.62 \mathrm{r} & 2.80 \mathrm{~h} & 23.13 & 23.17 & 22.07 & 22.79 & 41.60 \mathrm{t} & 44.17 \mathrm{qr} & 45.00 \mathrm{pq} & 43.591 & 2.09 \mathrm{q} & 2.19 \mathrm{p} & 2.37 \mathrm{n} & 2.22 \mathrm{i} & 43.50 \mathrm{pqr} & 42.20 \mathrm{rs} & 44.73 \mathrm{nop} & 43.48 \mathrm{i}\end{array}$

$\begin{array}{llllllllllllllllllllll}\text { T.-3 } & 2.35 \mathrm{~s} & 2.65 \mathrm{r} & 2.35 \mathrm{~s} & 2.45 \mathrm{i} & 23.13 & 23.07 & 22.00 & 22.73 & 41.00 \mathrm{t} & 41.00 \mathrm{t} & 41.80 \mathrm{st} & 41.27 \mathrm{k} & 1.79 \mathrm{~s} & 1.93 \mathrm{r} & 2.15 \mathrm{pq} & 1.96 \mathrm{k} & 37.80 \mathrm{t} & 40.97 \mathrm{~s} & 42.92 \mathrm{qr} & 40.56 \mathrm{k}\end{array}$

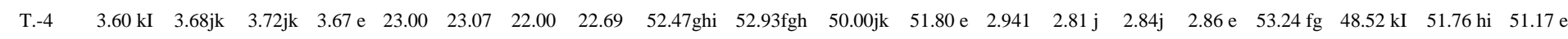

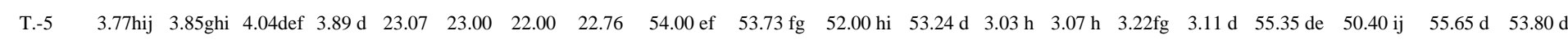

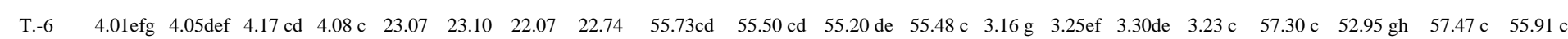

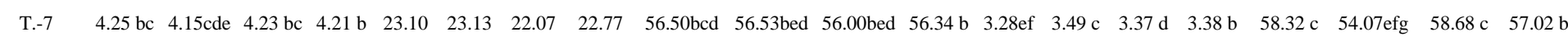

$\begin{array}{lllllllllllllllllllll}\text { T.-8 } & 3.91 \mathrm{fgh} & 3.48 \mathrm{Im} & 3.76 \mathrm{ij} & 3.72 \mathrm{e} & 23.13 & 23.07 & 22.00 & 22.73 & 46.83 \mathrm{mno} & 51.33 \mathrm{ij} & 49.20 \mathrm{kI} & 49.12 \mathrm{f} & 2.73 \mathrm{k} & 2.65 \mathrm{I} & 2.641 & 2.67 \mathrm{f} & 53.41 \mathrm{fg} & 46.00 \mathrm{mn} & 51.16 \mathrm{i} & 50.19 \mathrm{f}\end{array}$

$\begin{array}{lllllllllllllllllllll}\text { T.-9 } & 3.00 \mathrm{pq} & 3.24 \mathrm{n} & 3.43 \mathrm{~m} & 3.22 \mathrm{f} & 23.13 & 23.13 & 22.20 & 22.82 & 44.20 \mathrm{qr} & 47.07 \mathrm{mn} & 48.10 \mathrm{~m} & 46.46 \mathrm{~g} & 2.30 \mathrm{o} & 2.52 \mathrm{~m} & 2.55 \mathrm{~m} & 2.46 \mathrm{~g} & 47.171 \mathrm{~m} & 45.00 \mathrm{no} & 49.75 \mathrm{jk} & 47.31 \mathrm{~g}\end{array}$

\begin{tabular}{l|lllllllllllllllllllll} 
T.-10 & 2.88 q & 4.16 no & 2.98 pq & $3.01 \mathrm{~g}$ & 23.00 & 23.13 & 22.07 & 22.73 & $43.20 \mathrm{rs}$ & $45.57 \mathrm{opq}$ & $46.30 \mathrm{nop}$ & $45.02 \mathrm{~h}$ & $2.17 \mathrm{p}$ & $2.35 \mathrm{n}$ & $2.50 \mathrm{~m}$ & $2.34 \mathrm{~h}$ & $45.57 \mathrm{n}$ & $44.00 \mathrm{oq}$ & $47.00 \mathrm{~m}$ & $45.52 \mathrm{~h}$ \\
\hline
\end{tabular}

\begin{tabular}{|c|c|c|c|c|c|c|c|c|c|c|}
\hline LSD & CV.: - & \multirow{2}{*}{$\begin{array}{c}\text { T.: } \\
0.086\end{array}$} & CV.: -0.077 & \multirow{2}{*}{ T.: } & CV.: - 0.815 & \multirow{2}{*}{$\begin{array}{c}\text { T.: } \\
0.841\end{array}$} & CV.: -0.029 & \multirow{2}{*}{$\begin{array}{c}\text { T.: } \\
0.045\end{array}$} & CV.: -0.750 & \multirow{2}{*}{$\begin{array}{c}\text { T.: } \\
0.806\end{array}$} \\
\hline$(\mathrm{P} \leq 0.05)$ & CV. x T. : 0.1464 & & CV. x T. :- & & CV. x T. : 1.4669 & & CV. x T. : 0.0732 & & CV. x T. : 1.4062 & \\
\hline
\end{tabular}


Table 3. Yield components of wheat as affected by the removal of some photosynthetic organs in 2006.

\begin{tabular}{|c|c|c|c|c|c|c|c|c|c|c|c|c|c|c|c|c|c|c|c|c|}
\hline \multicolumn{5}{|c|}{ Spike weight (g) } & \multicolumn{4}{|c|}{ Number of spikelet per spike } & \multicolumn{4}{|c|}{ Number of grains/spike } & \multicolumn{4}{|c|}{ Grain weight/spike (g) } & \multicolumn{4}{|c|}{ 1000-grain weight (g) } \\
\hline \multirow[b]{2}{*}{ 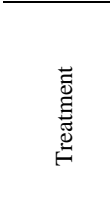 } & \multicolumn{3}{|c|}{ Cultivars } & \multirow[b]{2}{*}{ 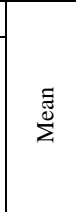 } & \multicolumn{3}{|c|}{ Cultivars } & \multirow[b]{2}{*}{ 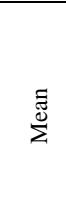 } & \multicolumn{3}{|c|}{ Cultivars } & \multirow[b]{2}{*}{ 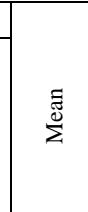 } & \multicolumn{3}{|c|}{ Cultivars } & \multirow[b]{2}{*}{ 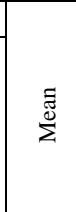 } & \multicolumn{3}{|c|}{ Cultivars } & \multirow[b]{2}{*}{$\begin{array}{l}\text { ॠ్ } \\
\sum^{\mathbb{2}}\end{array}$} \\
\hline & 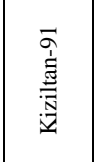 & 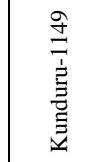 & 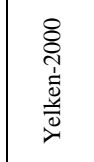 & & 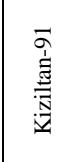 & 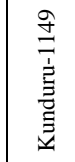 & 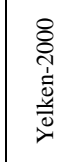 & & 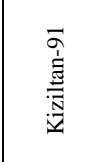 & 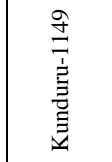 & 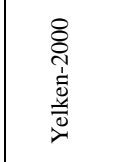 & & 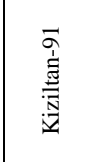 & 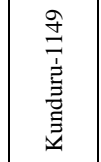 & 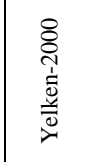 & & 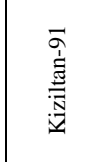 & 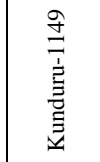 & 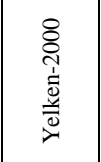 & \\
\hline T. -1 & 3.56e & $4.43 \mathrm{a}$ & $4.02 \mathrm{~b}$ & $4.01 \mathrm{a}$ & 22.13 & 23.40 & 21.83 & 22.46 & $52.93 \mathrm{~cd}$ & $56.20 \mathrm{ab}$ & 56.95 a & $55.36 \mathrm{a}$ & $2.60 \mathrm{e}$ & $3.41 \mathrm{a}$ & $3.21 \mathrm{~b}$ & $3.07 \mathrm{a}$ & $50.90 \mathrm{i}$ & $59.20 \mathrm{a}$ & $56.00 \mathrm{~cd}$ & $56.00 \mathrm{~cd}$ \\
\hline T.- 2 & $2.15 \mathrm{r}$ & $2.59 \mathrm{o}$ & 2.63 no & $2.45 \mathrm{~h}$ & 22.33 & 23.33 & 21.60 & 22.39 & $43.17 \mathrm{pq}$ & $43.00 \mathrm{pq}$ & $45.33 \mathrm{mn}$ & $43.83 \mathrm{i}$ & $1.64 \mathrm{r}$ & $2.14 \mathrm{~lm}$ & 2.02 no & $1.94 \mathrm{~h}$ & $41.50 \mathrm{r}$ & $50.13 \mathrm{i}$ & $44.90 \mathrm{no}$ & 44.90no \\
\hline T. -3 & $1.75 \mathrm{~s}$ & $2.40 \mathrm{p}$ & $2.30 \mathrm{q}$ & $2.15 \mathrm{i}$ & 22.20 & 23.37 & 21.73 & 22.43 & $41.83 \mathrm{qr}$ & $41.00 \mathrm{r}$ & 43.20 op & $42.01 \mathrm{k}$ & $1.38 \mathrm{~s}$ & $1.90 \mathrm{p}$ & $1.76 \mathrm{q}$ & $1.68 \mathrm{i}$ & $40.43 \mathrm{~s}$ & 45.30n & $36.10 \mathrm{t}$ & $36.10 \mathrm{t}$ \\
\hline T. -4 & $2.70 \mathrm{mn}$ & $2.91 \mathrm{i}$ & $2.90 \mathrm{ij}$ & $2.84 \mathrm{e}$ & 22.17 & 23.30 & 21.27 & 22.24 & $48.00 \mathrm{ij}$ & $47.50 \mathrm{ijk}$ & $51.47 \mathrm{ef}$ & $48.99 \mathrm{e}$ & $2.08 \mathrm{mn}$ & 2.41ghi & $2.40 \mathrm{ghi}$ & $2.30 \mathrm{e}$ & $45.57 \mathrm{mn}$ & 54.70 ef & $49.00 \mathrm{j}$ & $49.00 \mathrm{j}$ \\
\hline T. -5 & $2.84 \mathrm{jk}$ & $3.11 \mathrm{~h}$ & $3.31 \mathrm{~g}$ & $3.09 \mathrm{~d}$ & 22.33 & 23.60 & 21.67 & 22.53 & 49.83gh & $50.33 \mathrm{fg}$ & 52.43cde & $50.87 \mathrm{~d}$ & $2.23 \mathrm{kI}$ & $2.46 \mathrm{fg}$ & $2.53 \mathrm{ef}$ & $2.41 \mathrm{~d}$ & 46.471 & $56.30 \mathrm{c}$ & $52.20 \mathrm{~h}$ & $52.20 \mathrm{~h}$ \\
\hline T. -6 & 2.961 & $3.47 \mathrm{f}$ & $3.72 \mathrm{~d}$ & $3.38 \mathrm{c}$ & 22.47 & 23.37 & 21.20 & 22.34 & 51.80 de & 52.33cde & $53.33 \mathrm{c}$ & $52.49 \mathrm{c}$ & 2.41ghi & $2.57 \mathrm{e}$ & $2.81 \mathrm{~d}$ & $2.59 \mathrm{c}$ & $48.33 \mathrm{jk}$ & $57.83 \mathrm{~b}$ & $54.77 \mathrm{ef}$ & $54.77 \mathrm{ef}$ \\
\hline T. -7 & $3.05 \mathrm{~h}$ & $3.83 c$ & $3.84 \mathrm{c}$ & $3.57 \mathrm{~b}$ & 22.50 & 23.23 & 21.40 & 22.38 & $52.07 \mathrm{cde}$ & $54.90 \mathrm{~b}$ & $55.23 \mathrm{~b}$ & $54.06 \mathrm{~b}$ & $2.53 \mathrm{ef}$ & $2.76 \mathrm{~d}$ & $3.05 \mathrm{e}$ & $2.78 \mathrm{~b}$ & $49.13 \mathrm{j}$ & 58.67ab & 55.23de & 55.23de \\
\hline Т.-8 & $2.66 \mathrm{mn}$ & $2.86 \mathrm{j}$ & $2.85 \mathrm{j}$ & $2.79 \mathrm{e}$ & 22.50 & 23.43 & 21.73 & 22.56 & $47.33 \mathrm{jk} 1$ & $46.33 \mathrm{kIn}$ & $48.73 \mathrm{hi}$ & $47.47 \mathrm{f}$ & $2.15 \mathrm{Im}$ & $2.56 \mathrm{e}$ & $2.42 \mathrm{gh}$ & $2.38 \mathrm{~d}$ & 44.30op & $54.37 \mathrm{fg}$ & $48.43 \mathrm{jk}$ & $48.43 \mathrm{jk}$ \\
\hline T. -9 & $2.59 \mathrm{o}$ & $2.71 \mathrm{Im}$ & $2.78 \mathrm{kI}$ & $2.69 \mathrm{f}$ & 22.40 & 23.23 & 21.57 & 22.40 & $46.00 \mathrm{Im}$ & $45.00 \mathrm{mn}$ & $47.46 \mathrm{ijk}$ & $46.15 \mathrm{~g}$ & $2.02 \mathrm{no}$ & $2.36 \mathrm{hi}$ & $2.33 \mathrm{ij}$ & $2.24 \mathrm{f}$ & $43.47 \mathrm{pq}$ & $53.80 \mathrm{~g}$ & $47.97 \mathrm{k}$ & $47.97 \mathrm{k}$ \\
\hline T. -10 & $2.38 \mathrm{p}$ & $2.64 \mathrm{mno}$ & $2.70 \mathrm{mn}$ & $2.57 \mathrm{~g}$ & 22.57 & 23.30 & 21.47 & 22.44 & 44.53 no & 44.23nop & $46.33 \mathrm{kIm}$ & $45.03 \mathrm{~h}$ & $1.97 \mathrm{op}$ & $2.26 \mathrm{jk}$ & $2.20 \mathrm{kI}$ & $2.14 \mathrm{~g}$ & $42.67 \mathrm{q}$ & $52.60 \mathrm{~h}$ & $46.40 \mathrm{Im}$ & $46.40 \mathrm{Im}$ \\
\hline LSD & \multicolumn{3}{|c|}{ CV.: - 0.029} & \multirow{2}{*}{$\begin{array}{c}\left|\begin{array}{c}\text { T.: } \\
0.046\end{array}\right| \\
\end{array}$} & \multicolumn{3}{|c|}{ CV.: 0.482} & \multirow{2}{*}{ Т.: } & \multicolumn{3}{|c|}{ CV.: 0.727} & \multirow{2}{*}{$\begin{array}{c}\text { T.: } \\
0.781\end{array}$} & \multicolumn{3}{|c|}{ CV.: 0.037} & \multirow{2}{*}{$\begin{array}{c}\mathrm{T} .: \\
0.048\end{array}$} & \multicolumn{3}{|c|}{ CV.: 0.661} & T. \\
\hline$(\mathrm{P} \leq 0.05)$ & \multicolumn{3}{|c|}{ Т.: 0.0 .0} & & \multicolumn{3}{|c|}{ CV. x T. :- } & & \multicolumn{3}{|c|}{ CV. x T. : 1.3617} & & \multicolumn{3}{|c|}{ CV. $\times$ T. : 0.0896} & & \multicolumn{3}{|c|}{ CV. x T. : 0.8521} & 0.488 \\
\hline
\end{tabular}

$\mathrm{T}_{1}=$ Control(intaet plants). $\mathrm{T}_{2}=$ All leaf blades removed. $\mathrm{T}_{3}=$ All leaf blades and awns removed. $\mathrm{T}_{4}=$ Except flag leaf blade. other leaf blades removed, $T_{5}=$ Except flag leaf blade and l'tupper leaf blade, other leaf blades removed, $T_{6}=$ Except flag leaf blade, $1 \mathrm{t}$ upper leaf blade and 2nd upper leaf blade, other leaf blades removed, $T_{7}=$ Only awns removed. $T_{8}=$ Only flag leaf blade removed, $T_{9}=$ Flag leaf blade and $1^{\text {st }}$ upper leaf blade removed. $T_{10}=$ Flag leaf blade. $1^{\text {st }}$ upper leaf blade and $2^{\text {nd }}$ upper leaf blade removed. 


\section{Spike weight}

Considering the mean values of intact plants (control) in the experimental plots as $100 \%$, reduction rates of spike weight depending on the removal of some photosynthetic organs are shown in Fig. 1.

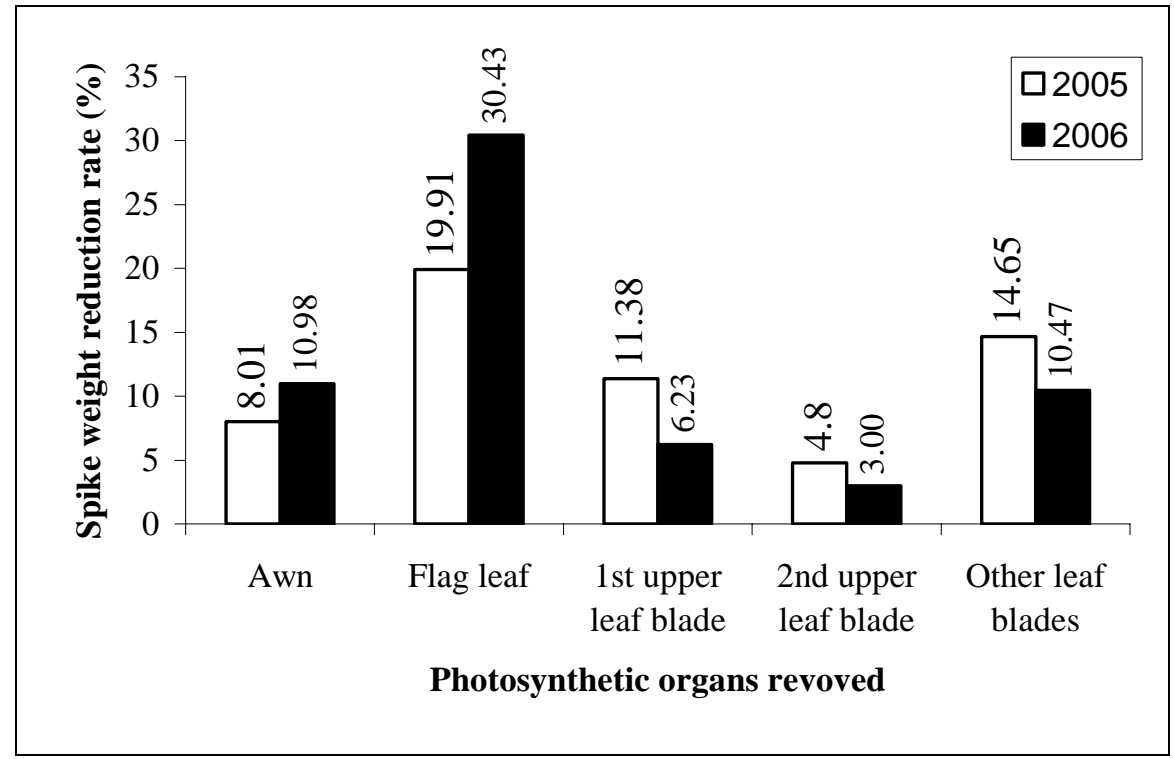

Fig. 1. Reduction rates of spike weight due to removal of some photosynthetic organs in 2005 and 2006.

In the first experimental year (2005), removal of awns, flag leaf blade, $1^{\text {st }}$ upper leaf blade, $2^{\text {nd }}$ upper leaf blade, and other leaf blades caused decrease in spike weight by $8.01 \%, 19.91 \%, 11.83 \%, 4.80 \%$, and $14.65 \%$, respectively (Fig. 1). In 2006, spike weight was reduced by $10.98 \%$ due to awns removal. Besides, the removal of flag leaf blade, $1^{\text {st }}$ upper leaf blade, $2^{\text {nd }}$ upper leaf blade and other leaf blades reduced spike weight by $30.43 \%, 6.23 \%, 3.00 \%$, and $10.47 \%$, respectively.

In 2006, crop received inadequate rainfall after heading. Older leaves of wheat plant dropped due to drought stress. Therefore, flag leaf photosynthesis occurred for longer time. Contribution of flag leaf to spike weight in 2006 was more than its contribution in 2005. Awns, a xeromorphic trait, performed more effectively in photosynthesis, especially in heat and drought conditions. Thus, their contribution to spike weight in 2006 was higher than that in 2005.

\section{Number of spikelets per spike}

According to results of variance analysis, effects of treatments on number of spikelets per spike were not statistically significant in both the years 2005 and 
2006 (Table 2 and 3). Therefore, change rates of number of spikelets per spike depending on some photosynthetic organs removal were not calculated.

\section{Number of grains per spike}

When the mean values of intact plants (control) in the experimental plots were considered as $100 \%$, reduction rates of number of grains per spike depending on some photosynthetic organs removal are shown in Fig. 2.

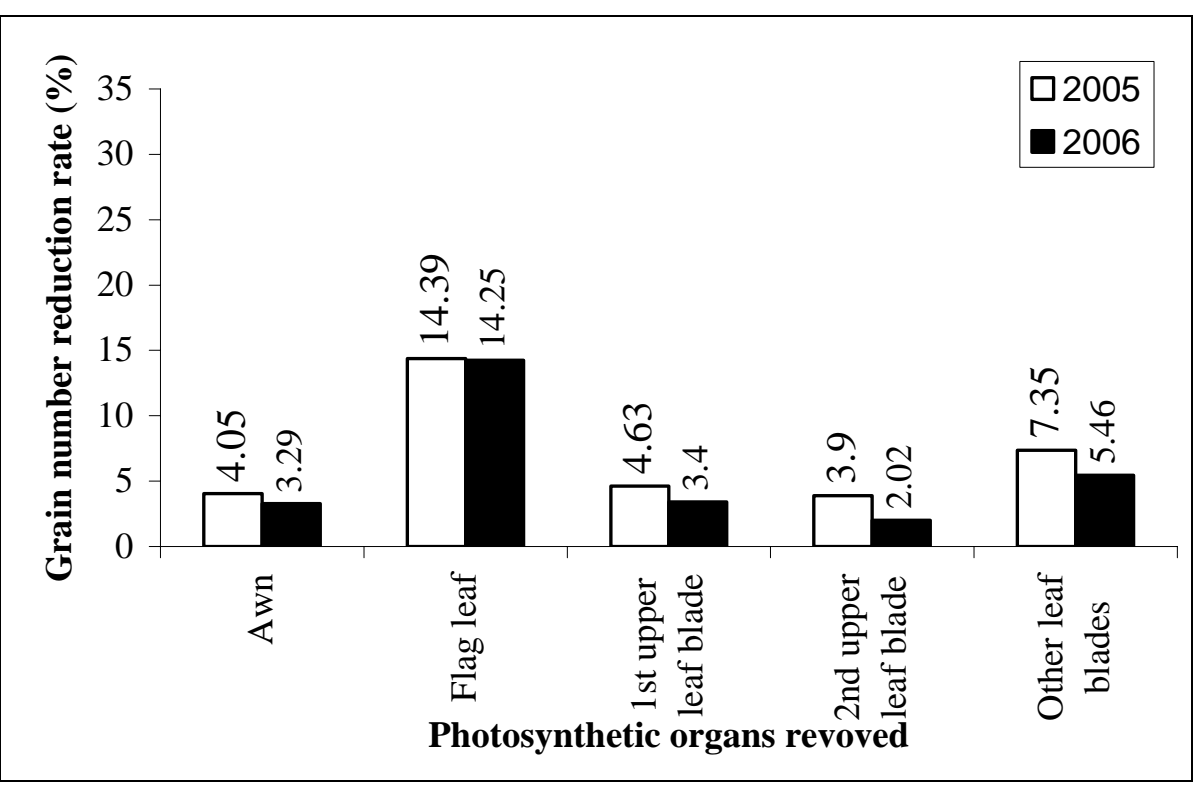

Fig. 2. Reduction rates of number of grains per spike due to removal of some photosynthetic organs in 2005 and 2006.

Fig. 2 shows that removal of awns reduced the number of grains per spike by $4.05 \%$ in 2005. Flag leaf blade, $1^{\text {st }}$ upper leaf blade, $2^{\text {nd }}$ upper leaf blade, and other leaf blades removal reduced the number of grain per spike by $14.39 \%$, 4.63, \%, $3.90 \%$, and $7.35 \%$, respectively, in 2005 during the year. In the second experimental year (2006), reduction rates of number of grains per spike due to removal of awns, flag leaf blade, 1 st upper leaf blade, $2^{\text {nd }}$ upper leaf blade and other leaf blades were $3.29 \%, 14.25 \%, 3.40 \%, 2.02 \%$ and $5.46 \%$, respectively.

\section{Grain weight per spike}

As mean values of intact plants (control) in the experimental plots were accepted as $100 \%$, reduction rates of grain weight per spike depending on some photosynthetic organs removal are shown in Fig. 3. 


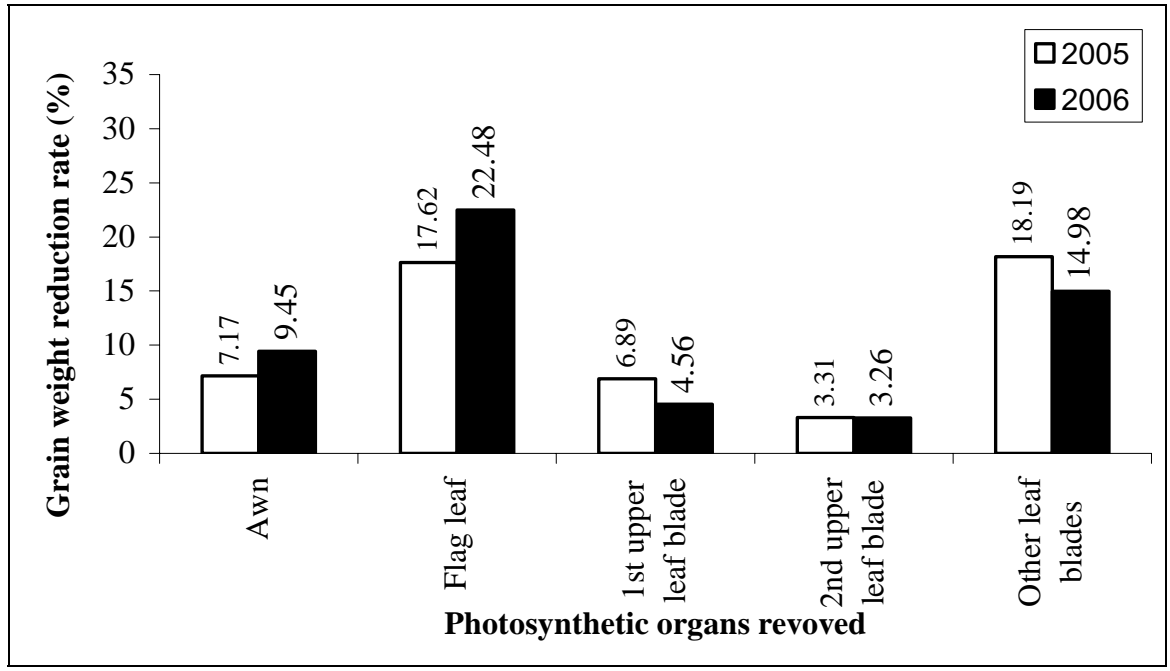

Fig. 3. Reduction rates of grain weight per spike due to removal of some photosynthetic organs in 2005 and 2006.

Based on the results of first year (2005), due to awns removal reduction rate of grain weight per spike was $7.17 \%$ (Fig. 3). Flag leaf blade removal caused a reduction of $17.62 \%$ in grain weight per spike. As $1^{\text {st }}$ upper leaf blade, $2^{\text {nd }}$ upper leaf blade and other leaf blades were removed, grain weight per spike was reduced by $6.89 \%, 3.31 \%$, and $18.19 \%$, respectively. According to results of the second year (2006), grain weight per spike was reduced by $9.45 \%$ due to awns removal. As flag leaf blade was removed, reduction rate of grain weight per spike was $22.48 \%$. Besides, removal of $1^{\text {st }}$ upper leaf blade, $2^{\text {nd }}$ upper leaf blade, and other leaf blades caused reduction of grain weight per spike by $4.56 \%, 3.26 \%$, and $14.98 \%$, respectively.

As the rainfall was higher in 2005 than in the fallowing year at heading stage, the contribution of awns and flag leaf blade to grain weight per spike was higher in 2006.

\section{0-grain weight}

As mean values of intact plants (control) in the experimental plots were considered as $100 \%$, reduction rates of 1000 -grain weight depending on some photosynthetic organs removal are shown in Fig.e 4. 


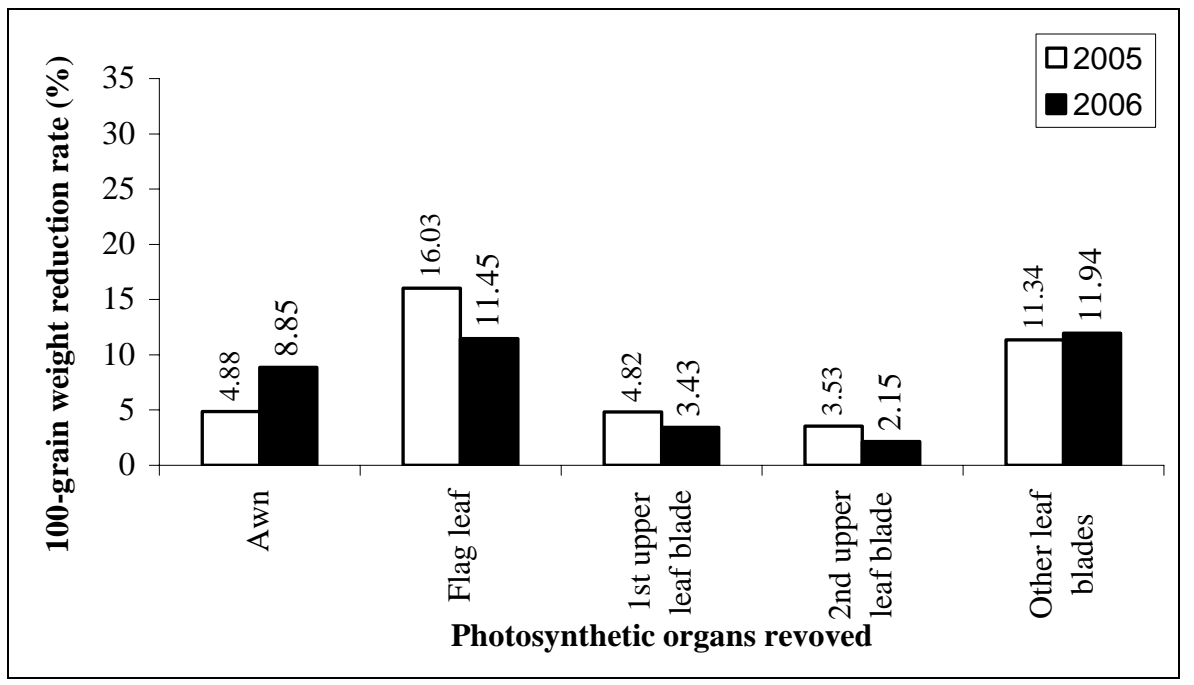

Fig. 4. Reduction rates of 1000-grain weight due to removal of some photosynthetic organs in 2005 and 2006.

As seen in the Fig. 4, removal of awns, flag leaf blade, $1^{\text {st }}$ upper leaf blade, $2^{\text {nd }}$ upper leaf blade and other leaf blades resulted in the decrease in 1000-grain weight by $4.88 \%, 16.03 \%, 4.82 \%, 3.53 \%$, and $11.34 \%$, respectively, in 2005 . 1000 -grain weight was reduced by $8.85 \%$ due to awns removal in 2006. Due to the removal of flag leaf blade, 1st upper leaf blade, 2 upper leaf blade and other leaf blades, 1000-grain weight was reduced by $11.45 \%, 3.43 \%, 2.15 \%$ and $11.94 \%$, respectively during the year. Contribution of awns to 1000 -grain weight in 2006 was higher than that in 2005 since rainfall was less in 2006 at heading stage.

\section{Discussion}

The results of this study showed that removal of awns, flag leaf blade, $1^{\text {st }}$ upper leaf blade, $2^{\text {nd }}$ upper leaf blade significantly reduced main yield components of durum wheat. These organs are the most important photosynthetic organs for wheat. Their contribution rates to main yield components vary depending on both ecological conditions and genotypes. Besides, the awns on main yield components are very effective under drought stress, especially at heading stage. Kriedemann (1966) pointed out that the contribution made by ear photosynthesis to grain yield was found to vary from $10 \%$ to $44 \%$ depending on the technique used and environmental conditions. It was reported that a large amount of awns in the ear was a drought adaptive attribute in cereals (Blum, 1985). Olugbemi and Bush (1987) stated that awns increased photosynthetic rates of the ears. Araus et al. (1993) found that in durum wheat, if flag leaves (blade plus sheath) and ears 
were kept in the dark from one week after anthesis to maturity, grain weight was reduced by $22.4 \%$ and $59.0 \%$, respectively. Mahmood and Chowdhry (1997) emphasized that removal of flag leaf resulted in $16.4 \%, 14.8 \%$, 34.5\%, and 20.0 $\%$ reduction in seed set percentage, number of grains per spike, grain weight per spike, and 1000-grain weight, respectively. Reduction in these traits as a consequence of the removal of $3^{\text {rd }}$ nodal leaf and awns was also significant. Katsileros et al. (2002) reported that the removal of awns reduced yield and its components significantly, while the removal of the flag leaf had the same effect but of smaller magnitude. The $50 \%$ reduction in the number of kernels per spike increased significantly the 1000-kernel weight which, however, did not prevent a reduction in total yield. Motzo and Giunta (2002) found that awns increased the ear surface from $36 \%$ to $59 \%$, depending on their length. Awns positively affected grain yield, with an average increase of 10 and $16 \%$. The effects of awns on grain yield and kernel weight strongly depend on the genetic background, on awn length and environmental conditions during grain filling. Birsin (2005) raported that grain number per spike was reduced by $12.4 \%$ to $12.8 \%$ as the flag leaf was removed. It was also reduced by $5.1 \%$ to $5.5 \%$ with the removal of second upper leaf blade. Besides, as awns were removed, grain weight per spike was reduced by $8.8 \%$ to $13.1 \%$ and it was reduced by $4.4 \%$ to $8.7 \%$ with the removal of second upper leaf blade. Bhutta (2006) pointed out that flag leaf area that had significant correlation with grain yield could be used as selection criteria to identify drought-tolerant wheat genotypes. Li et al. (2006) stated that in wheat, the leaves particularly the flag leaves have been considered to be the key organs contributing to higher yields. Besides, their results suggested that awns play a dominant role in contributing to large grains and high grain yield in awned wheat cultivars, particularly during the grain filling stage.

\section{Conclusion}

A review of the results has made it clear that flag leaf blade, $1^{\text {st }}$ upper leaf blade and $2^{\text {nd }}$ upper leaf blade were the most important photosynthetic organs of durum wheat during the grain filling stage. Besides, awns performed an effective role in photosynthesis, especially under the drought conditions. Spike weight, number of grains per spike, grain weight per spike, and 1000-grain weight were significantly reduced due to removal of these photosynthetic organs in wheat. It is concluded that the flag leaf should be utilized as an important morphological trait to increase yield in durum wheat.

\section{References}

Araus, J. L., H. R. Brown, A. Febrero, J. Bort and M. D. Serret, 1993. Ear photosynthesis, carbon isotope discrimination and the contribution of respiratory 
$\mathrm{CO}_{2}$ to differences in grain mass in durum wheat. Plant, Cell \& Environment 16 (4): 383-392.

Austin, R. B. and H. G. Jones, 1975. The physiology of wheat. Annual Report of The Plant Breeding Institute for 1975, Trumpington, 20-73.

Bhutta, W. M., 2006. Role of some agronomic traits for grain yield production in wheat ( Triticum aestivum L.) genotypes under drought conditions. Revista UDO Agricola 6(1): 11-19.

Birsin, M. A., 2005. Effects of removal of some photosynthetic structures on some yield components in wheat. Ankara University, Faculty of Agriculture. Journal of Agricultural Science 11(4): 364-367.

Blum, A. 1985. Photosynthesis and transpiration in leaves and ears of wheat and barley varieties. Journal of Experimental Botany 36 (3): 432-440.

Duzgune, 0., T. Kesici, 0. Kavuncu, and F. GurbUz. 1987. Aratirma ye Deneme Metotlari (Istatistik Metodlari II). AU. Ziraat FakUltesi Yayinlari: 1021. Ders Kitabi, 295 s. Ankara (in Turkish).

Evans, L. T. and M. Rawson, 1970. Photosynthesis and respiration by the flag leaf and components of the ear during grain development in wheat. Aust. J. Biol. Sci. 23: 245254.

Genç, I, 1977. Physiological and morphological principles of grain yield in cereals. Ç.U. Annal of Agricultural Faculty 8:1.

Jiang, Q. Z., D. Roche, S. Durham and D. Hole. 2006. Awn contribution to gas exchanges of barley ears. Photosynthetica 44 (4): 536-541.

Katsileros, A. D., P. J. Kaltsikes and P. J. Bebeli. 2002. Effect of flag leaf, the awns and the number of kernels per spike on grain yield and its components in durum wheat (Triticum turgidum L. var. durum). Agricultural Research 25 (2): 15-20.

Koç, M., H. Winzeler und J. Nösberger, 1985. Die $\mathrm{C}_{2}$-Austauschrate der Ahre und die Verteilung der Aehrenassimilate von zwei. Sommer weizensorten, Schweiz. Landw. F, 24, $1 / 2,57-67$.

Kridemann, P. 1966. The photosynthetic activity of the wheat ear. Annals of Botany 30: 349-363.

Li, X., H. Wang, H. Li, L. Zhang, N. Teng, Q. Lin, J. Wang, T. Kuang, Z. Li, B. Li, A. Zhang and J. Lin. 2006. Awns play a dominant role in carbohydrate production during the grain-filling stages in wheat (Triticum aestivum L.). Physiologia plantarum 127 (4): 701-709.

Mahmood, N. and M. A. Chowdhry. 1997. Removal of green photosynthetic structures and their effect on some yield parameters in bread wheat. Wheat Information Service 85: 14-20.

Migus, W. N. and L. A. Hunt, 1980. Gas exchange rates and nitrogen concentrations in two winter wheat cultivars during the grain filling period. Can. J. Bot. 58: 21 102116. 
Motzo, R. and F. Giunta. 2002. Awnedness affects grain yield and kernel weight in nearisogenic lines of durum wheat. Australian Journal of Agricultural Research 53 (12): 1285-1293.

Olugbemi, L. B. and M. G. Bush. 1987. The influence of temperature on the contribution of awns to yield in wheat. Australian Journal of Plant Physiology 14 (3): 299-310.

Steel, R.G.D. and J.H. Torrie. 1984. Principles and procedures of statistics. Mc. Graw Hill Book Co. Inc., New York.

Thorne, N. G., 1973. Physiology of grain yield of wheat and barley. Rothamsted Experimental Station Report for 1973. (Part 2).

Weyhrich, R. A., B. F. Carver and B. C. Martin. 1995. Photosynthesis and water-use efficiency of awned and awnletted near-isogenic lines of hard winter wheat. Crop Sci 35: 172-176.

Zadoks, J.C., T.T. Chang, and C.F. Konzak. 1974. A decimal code for growth stages of cereals. Weed Res. 14: 415-421. 